MEIOSIS

\section{Under arrest}

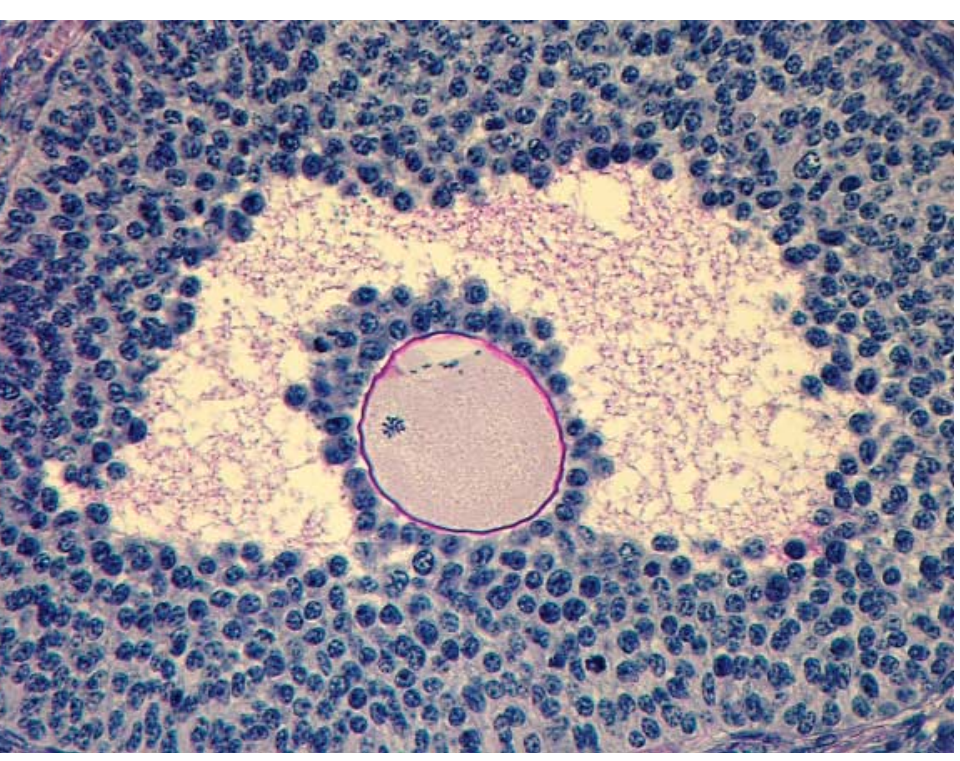

An oocyte with a polar body within an antral follicle of a $\mathrm{Gpr}^{-/-}$ovary. Image kindly provided by Laurinda J affe, University of Connecticut Health Center, Farmington, CT, USA.
M ammalian oocytes are arrested in prophase of meiosis from a time before birth. Then, during reproductive life, luteinizing hormone ( $\mathrm{LH})$ induces the resumption of meiosis, which results in a haploid set of chromosomes in preparation for fertilization. Researchers reporting in Science havenow shed light on how meiotic arrest is maintained in mouse oocytes up until the time that $\mathrm{LH}$ induces the resumption of meiosis and ovulation.

The heterotrimeric $\mathrm{G}$ protein $\mathrm{G}_{\mathrm{s}}$ had previously been shown to be required for meiotic arrest, so M ehlmann and Jaffe, in collaboration with Eppig and colleagues, searched a database of genes that are expressed in mouse oocytes, looking for a receptor that activates $G_{s^{\prime}}$. The search identified the orphan $G_{s}$-linked receptor GPR3, which increases cyclic-AM P concentrations. This was an interesting result because $G_{s}$ is known to keep CAM P concentrations elevated, which isimportant for meiotic arrest. N ext, they showed that
Gpr3 mRNA was present in oocytes, and that the concentration of G pr3 transcripts was 14 timeshigher than in the surrounding somatic cells of antral (or resting) follicles.

To probe the function of GPR3, Mehlmann, Jaffe and co-workers analysed the ovaries of Gpr3-knockout mice. Theovaries were normal in terms of their external morphology, growth and ovulatory activity. H owever, $82 \%$ of the oocytes within antral Gpr3-/- follicles had resumed meiosis, and almost half of thesewere in metaphase II. Only $37 \%$ of oocytes from early antral Gpr3-1- follicles resumed meiosis, whereas all oocytes in preantral $\mathrm{Gpr}^{-1-}$ follicles remained arrested in prophase. So, depending on the developmental stage of the follicles, oocytes that lacked GPR3 resumed meiosis.

Next, the authors showed that the resumption of meiosisin $\mathrm{Gpr}^{-/-}$follicles was independent of $\mathrm{LH}$. The action of LH would normally lead to the deposition of extracellular material around the somatic granulosa cells,

\section{SIGNALLING}

\section{A pick-me-up}

Src becomes activated in response to various extracellular stimuli, but the hows and wheres have been somewhat hazy. Thanks to the use of a Src fusion protein, Sandilands et al. havenow found that Src becomes activated during transit from the perinuclear region to the plasma membrane. The actin cytoskeleton is required for this, as are RhoBassociated cytoplasmic endosomes.

Once the authors had established that the green fluorescent protein (GFP) tag that they engineered onto Src (to make Src- GFP) didn't interfere with the normal spatial distribution, activation or interactions of Src, they continued with themain aim of their studies to address the connection between catalytic activation and spatial localization. They saw that Src activity increased from the perinuclear region, where it was inactive, to peripheral membranestructures, where it was highly active. Artificially disrupting actin filaments inhibited both themembrane translocation and activity of Src. And when a mutant version of suppressor of CAR (SCAR)/WAVE1 was used to inhibit actin nucleation, Sandilands et al. showed that Src-GFP couldn't translocate to the plasma membrane in response to stimulation by platelet-derived growth factor (PDGF). This hinted that 'outside-in' signals from the PDGF receptor that induce thetranslocation/ activation of Src required signalling to the actin-polymerization machinery.

But the results didn't preclude the idea that endosomes - actin-associated structures with which Src has previously been reported to colocalize - might be involved in this 'outside-in' signalling. Indeed, the authors showed that active Src- GFP colocalized with RhoB, an endosome-associated Rho GT Pase, in the perinuclear region and in discrete intracellular structures that surround this region. In the absence of RhoB, Src failed to accumulate at the membrane or be activated in response to cell plating on fibronectin (a main Src-activating extracellular stimulus).

So, could there be a connection between these endosomal structures and the actinpolymerization machinery? Immunostaining showed that Src- GFP and fluorescently tagged forms of RhoB and SCAR/WAVE 1 all localized to discrete cytoplasmic structures. Disrupting actin filaments didn't alter RhoB endosomesfrom cells that lacked Src and the Src-family proteins Fyn and Yes - that is, until Src was re-expressed, in which case
RhoB endosomes remained in the perinuclear region. This implies that the presence of Src in RhoB endosomes somehow resulted in them being dependent on the actin cytoskeleton to move. When Src was induced to move to the peripheral membraneat the sametimeas actin polymerization was re-initiated (both by external means), motile Src- and RhoBcontaining structures that resembled endosomal vesicles were associated with newly formed small bundles of actin - actin clouds - between the perinuclear region and the cell membrane. The actin clouds weren't seen when the mutant SCAR/WAVE1 construct was expressed, implying that Src is promoting the actin-nucleation events that are associated with the endosomes.

The authors, therefore, postulate the following model: inactive Src is usually present around the perinuclear region, from where it is'picked up' and transported to peripheral membranes in RhoB-associated, Rabll-dependent endosomes, probably by inducing actin-nucleation events. D etails of exactly how Src induces these actin-nucleation events are awaited.

Katrin Bussell

(2) References and links ORIGINAL RESEARCH PAPER Sandilands, E. et al. RhoB and actin polymerization coordinate $\mathrm{Src}$ activation with endosome-mediated delivery to the membrane. Dev. Cell 7 855-869 (2004) 
